Nonlinear Processes in Geophysics (2005) 12: 83-88

SRef-ID: $1607-7946 / n p g / 2005-12-83$

European Geosciences Union

(c) 2005 Author(s). This work is licensed

under a Creative Commons License.

\title{
On the separation of timescales in spring-block earthquake models
}

\author{
S. Hergarten and F. Jansen \\ Institute of Geology, University of Bonn, Germany \\ Received: 12 July 2004 - Revised: 30 November 2004 - Accepted: 6 January 2004 - Published: 25 January 2005 \\ Part of Special Issue "Seismicity pattern dynamics"
}

\begin{abstract}
One of the most widespread spring-block earthquake models, the Olami-Feder-Christensen model, is investigated without making the assumption that the duration of individual earthquakes is negligible. While the GutenbergRichter law for the size distribution of earthquakes is preserved qualitatively for earthquakes of finite duration, the $b$ value decreases with increasing earthquake duration. The effect decreases with increasing lattice size, although it is not clear whether it completely vanishes in the limit of infinite grid size. For realistic values of earthquake duration, the effect is negligible, so that the original model with zero earthquake duration is appropriate for most applications.
\end{abstract}

\section{Introduction}

Spring-block earthquake models have a long history in seismology. Models of this type were designed as a simplified analogon to a fault (or a fault system). They consist of an array of masses which are connected with each other by elastic springs (Fig. 1). In addition, each mass is connected to a rigid plate by a leaf spring. This plate is responsible for long-term driving of the system, i.e., for a slow increase of the forces acting on each mass. In most models, this plate is assumed to move at a given constant velocity, corresponding to a constant rate of displacement at the fault. It is assumed that each mass sticks at the ground as long as the force acting on it (through all springs) are below a given threshold. If the force on any mass exceeds this threshold, it becomes unstable, and displacement occurs. As a result, the forces acting on other masses may change, so that further masses may become unstable. This may lead to some kind of avalanches which are interpreted as earthquakes of different sizes.

The first model of this type was proposed by Burridge and Knopoff (1967); it describes the stick-slip motion of a onedimensional chain of masses. The displacement of unstable

Correspondence to: S. Hergarten

(hergarten@geo.uni-bonn.de) masses was computed by a set of coupled ordinary differential equations. Several constitutive laws for the motion of unstable blocks have been proposed, e.g. constant or velocitydependent friction (Carlson et al., 1994; Turcotte, 1997).

However, as soon as a large number of masses is involved in an earthquake, the numerical effort becomes high because a large set of coupled equations must be solved. Therefore, a major step towards simulating large earthquake statistics was made by introducing simplified models which do not simulate the motion of individual masses in detail but use simpler rules. The simplification hinges on the fact that the duration of an individual earthquake is much shorter than time spans between earthquakes, so that finally the time scale of longterm driving can be separated from the time scale of individual earthquakes. In principle, this means that each earthquake shrinks to a point on the time axis or, in other words, the motion of the driving plate stops during an earthquake. A first step in this direction was performed by Rundle and Jackson (1977), several others followed (Nakanishi, 1990; Brown et al., 1991; Matsuzaki and Takayasu, 1991; Olami et al., 1992). At this time, the interest in spring-block models was renewed because their relationship to the theoretical concept of self-organized criticality (SOC) (Bak et al., 1987; Bak, 1996; Jensen, 1998; Hergarten, 2002) was discovered.

Although separating the timescales of rupture propagation and earthquake sequences seems to be straightforward, there is still discussion on the separation of earthquakes. Rubin (2002) and Vidale et al. (2003) argue that there is a gap of about $10 \mathrm{~s}$ between the duration of an earthquake and the beginning of the aftershock sequence. In contrast, Kagan and Knopoff (1981) and Kagan (2004) suggest that the same mechanism describes the propagation of an earthquake and cascades of aftershocks, so that the definition of an earthquake is rather arbitrary. From this the question arises to which extent statistical properties of earthquake sequences are affected by the duration of individual earthquakes and its influence on separating earthquakes. 


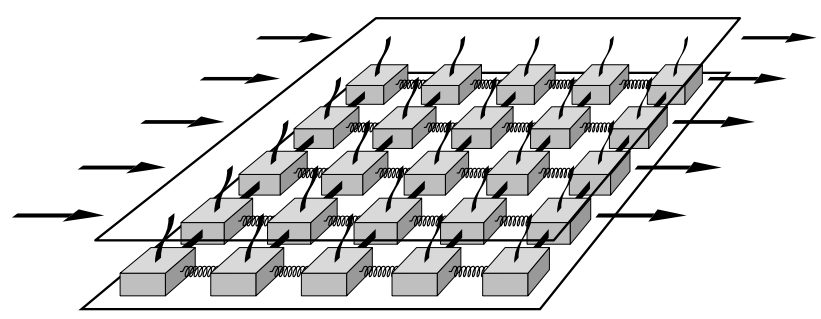

Fig. 1. Setup of a spring-block earthquake model.

\section{Olami-Feder-Christensen model}

In the following we focus on the The Olami-FederChristensen (OFC) model (Olami et al., 1992), which is one of the most widespread spring-block models in seismology as well as one of the most studied models in the field of SOC. The OFC model was the first to combine a striking similarity to earlier SOC models with the ability to reproduce the Gutenberg-Richter law (Gutenberg and Richter, 1954) for the size distribution of earthquakes.

The OFC model refers to a two-dimensional, square array of masses as shown in Fig. 1 and establishes the following rules:

Long-term driving: As a result of moving the driver plate, the total force acting on each mass (the sum of the forces exerted by the five adjacent springs) increases uniformly through time.

Relaxation: As soon as the total force acting on any mass reaches a given threshold, the mass becomes unstable, initiating an earthquake. The unstable mass is immediately displaced to its equilibrium position where its total force is zero. As a result, the forces acting on adjacent masses have increased, so that some of them may have become unstable, too. These masses are relaxed according to the same rule, instantaneously and independently of each other. If further masses have become unstable then, the procedure is repeated until the system has come to rest.

Let us use nondimensional variables in such a way that both the rate of driving and the threshold of instability are unity. Let us further assume that all horizontal springs are of equal strengths (no difference between helical and leaf springs), and that $k$ denotes the ratio of the elastic constant of the vertical leaf springs to that of the horizontal springs. Using a single index $i$ for numbering the masses, the OFC model can then be written as a simple cellular automaton with the variables $F_{i}$ as the forces according to the flow chart shown in Fig. 2.

The inner loop describes the stages of an individual earthquake, while the outer loop simulates a sequence of earthquakes. The relaxation rule for unstable masses is rather simple: A certain fraction $\alpha_{i}=\frac{1}{v_{i}+k}$ (where $v_{i}$ is the number of nearest neighbors of $i$ ) of the relaxed force is transferred to each of the nearest neighbors, while the rest, amounting to $1-v_{i} \alpha_{i}=\frac{k}{v_{i}+k}$, is returned to the driver plate and thus lost.

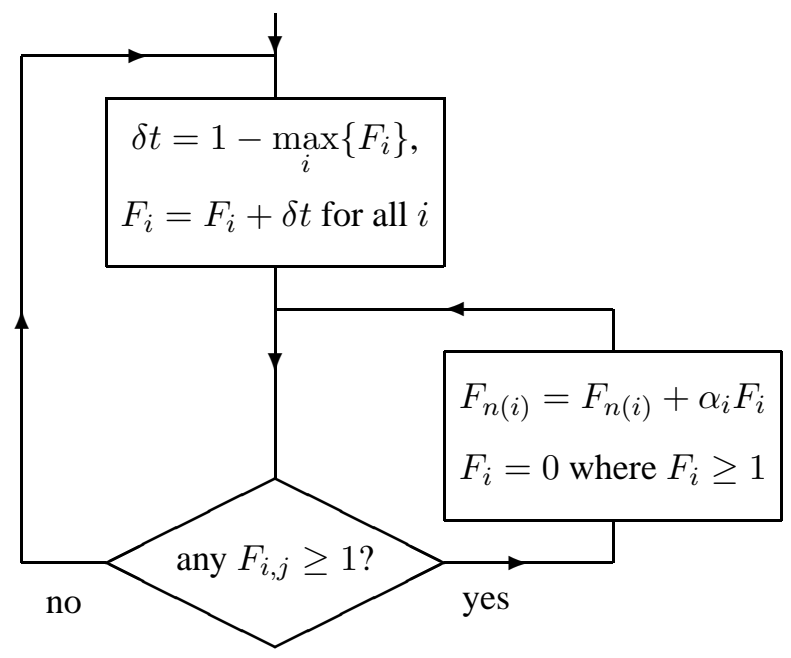

Fig. 2. Flow chart of the OFC model. The abbreviation $n(i)$ represents all nearest neighbors of the mass $i ; \alpha_{i}=\frac{1}{v_{i}+k}$ where $v_{i}$ is the number of nearest neighbors of $i$.

This means that the OFC model is nonconservative for $k>0$, in contrast to the most widespread SOC model, the sandpile model (Bak et al., 1987). OFC studied the nonconservative regime in detail and found that the model shows SOC not only in the conservative limiting case $k=0$, but also in the dissipative regime $k>0$. They also found that the power-law exponent of the event size distribution increases with $k$, i.e. as the level of conservation decreases, and that exponents consistent with the Gutenberg-Richter law (Gutenberg and Richter, 1954) found for earthquakes in nature are achieved if all springs have roughly the same strength $(k \approx 1)$.

Recent results have renewed the discussion on the OFC model. From the view of application, the discovery of foreshocks and aftershocks obeying Omori's law may be interesting (Hergarten and Neugebauer, 2002). It was recently found that several further properties of foreshock and aftershock sequences are reproduced by the model at least qualitatively, although clustering of earthquakes is too weak compared to real seismicity (Helmstetter et al., 2004). These results make the OFC model attractive for application in seismology, but some other results raise doubts concerning its applicability to real earthquakes. As reviewed by Jensen (1998), the OFC model was found to be not robust against quenched disorder, which is incommensurate to the inhomogeneity of the earth's crust. Furthermore, it has recently been claimed that many results obtained in the nonconservative regime may not hold in the limit of infinite system size and infinite simulation time. Lise and Paczuski (2001a,b) found that the power-law exponent may not increase with decreasing level of conservation but may be universal at least over a certain range of parameter values. Drossel (2002) observed that the event size distribution depends on the numerical accuracy in the strongly nonconservative regime and suspected that all earlier results might be artefacts of numerical inaccuracies. This topic is discussed in view of our results later in this paper. 


\section{Procedure}

We now return to a version of the OFC model with finite earthquake duration, which means that we skip the separation of time scales between individual events and long-term driving. In the context of basic SOC models, the first systematic study in this direction was published by Corral and Paczuski (1999). This study referred to a one-dimensional sandpile model, the "Oslo" model. It was found that, depending on the duration of individual events, avalanche merging occurs which finally turns into continuous flow. A version of the OFC model with finite event duration was considered by Hamon et al. (2002) in the context of solar flare statistics.

Compared to the original OFC model with instantaneous relaxation, we modify the model rules in the following way: The first mass involved in an earthquake is instantaneously relaxed, but before it is checked whether further masses have become unstable, model time is increased by a given increment $\tau$. The increment $\tau$ corresponds to the time span needed for the relaxation of one mass and is considered as a parameter or, in other words, the time needed for rupture to proceed from one mass to an adjacent mass. We therefore denote $\tau$ "rupture propagation time" in the following.

If further masses have been destabilized (these may be more than in the original model since all forces have increased by $\tau$ ), these masses are simultaneously relaxed as in the OFC model. It is again assumed that the relaxation takes the time $\tau$ (not for each mass, but for all masses which are relaxed simultaneously). After increasing $t$ by $\tau$, stability is checked again and unstable masses are relaxed. This procedure is repeated until the system has come to rest again. Then we switch to the next earthquake by increasing $t$ up to the point where the next mass becomes unstable. Modifying the flow chart (Fig. 2) is simple, we must only add the action $t=t+\tau$ to the box on the right-hand side (within the loop simulating an individual earthquake). The original OFC model is recovered for $\tau=0$.

This procedure is nearly the same as that suggested by Hamon et al. (2002) for modeling solar flare statistics. The only difference concerns long-term driving between events. While our approach preserves the continous driving rule of the OFC model, their approach introduces driving in discrete portions $\tau$.

Extending the algorithm suggested by Grassberger (1994) for efficient simulation of the model (for a detailed description see, e.g. Hergarten, 2002) is straightforward, so that the modified model can be simulated as efficiently as the original OFC model. However, analyzing the behavior in dependence of the parameter $\tau$ requires a high computational effort since a large sequence of events must be simulated for each value of $\tau$ in order to obtain reliable statistics. We therefore use a simplified approximation to this model, based on simulations of the original OFC model. First, the original model is simulated until transient components have vanished in order to achieve a quasi-steady state. Then, a sequence of $10^{9}$ events is computed and stored, including time of occurrence $t_{i}$, size $s_{i}$, and number of relaxation cycles $c_{i}$ (number of runs of the inner loop in Fig. 2) needed until the system comes to rest again for each event $i$. In a second step, a value of the parameter $\tau$ is chosen, and it is assumed that the duration of each event is $\tau c_{i}$, so that this event ends at the time $e_{i}=t_{i}+\tau c_{i}$. If the next event starts before this one has ended, i.e. if $t_{i+1} \leq e_{i}$, it is assumed that both events are not separated. In this case, the two events $i$ and $i+1$ are joined to one new event which starts at the time $t_{i}$, ends at the time $\max \left\{e_{i}, e_{i+1}\right\}$ and has the size $s_{i}+s_{i+1}$. This procedure is applied to the following events, too, so that a set of events is joined if they overlap in time.

The main advantage of this approximation is that it can be applied for different values of $\tau$ by reanalyzing earthquake sequences of the original OFC model without running the model. In return, we cannot measure event sizes in terms of the number of masses involved in the event (the rupture area), but only in terms of the number of relaxations. The reason is that the number of relaxed blocks is not additive if the rupture areas of different events overlap. However, it turns out that this practically makes no difference since the probability of overlapping rupture areas is very low if $\tau$ is not too large in the nonconservative OFC model.

The model is run with free boundary conditions, in contrast to several earlier studies where rigid-frame boundaries were used. For free boundary conditions, the parameter $\alpha_{i}$ is higher at the boundaries than in the bulk since the actual number of neighbors is only two or three instead of four. As discussed by Hergarten (2002), transient periodic components die out more rapidly under free boundaries, so that the quasi-steady state required for a stable statistics is achieved earlier. Apart from a slightly smaller exponent of the earthquake size distribution (for given values of $k$ ), free boundary conditions apparently have no further effect on the results.

\section{Results}

Figure 3 shows the event size statistics obtained from the simple approximation where the finite rupture propagation time $\tau$ is introduced afterwards. The parameter $k$ is chosen to $k=1$, which is the most widely used value for the OFC model. The lattice consists of $512 \times 512$ masses. The statistics are derived from a sequence of $10^{9}$ events, while a much larger number of events was skipped in the beginning of the simulation in order to avoid artefacts of transient components. The event size $s$ is measured in terms of the number of relaxations (if a mass is displaced more than once, it is counted more than once, too), $n(s)$ is the number of events of size $s$.

The effect of the rupture propagation time $\tau$ becomes visible for $\tau \geq 2 \times 10^{-6}$. While the power law is preserved qualitatively, its exponent decreases. For comparison, the dashed line gives the result obtained from simulating $10^{8}$ events with the full model for $\tau=10^{-5}$. The curves of both model versions are parallel; this shows that the approximation preserves the behavior of the full model. The shift between the curves arises from the fact that the $10^{9}$ events taken from the 


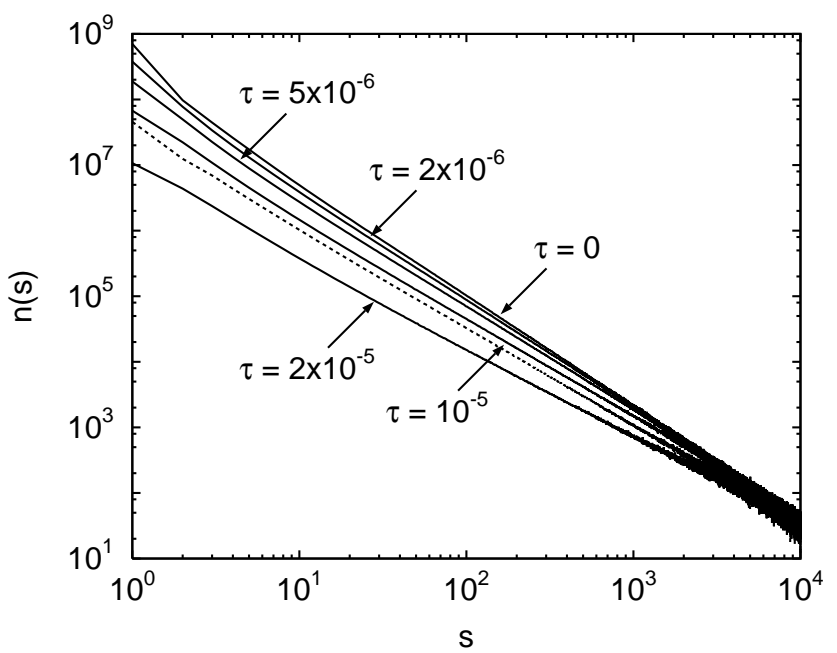

Fig. 3. Event size statistics obtained from the simplified model. The dashed line gives the result from the full model and $\tau=10^{-5}$ for comparison.

original OFC model collapse to $1.5 \times 10^{8}$ events for $\tau=10^{-5}$, while only $10^{8}$ events were simulated with the full model.

For a more quantitative view, we determine the exponents $b$ of the obtained power-law size distributions $P(s) \sim s^{-b}$. In order to keep the analogy to the established GutenbergRichter law, the distribution is considered in the cumulative sense, so that $P(s)$ is the probability that the size of an arbitrary event is greater or equal to $s$, although in the physics literature noncumulative distributions are often preferred. Technically, the exponent $b$ is obtained from fitting a power law to the noncumulative data in order to avoid artefacts of fitting cumulative distributions. The range of fitting is from 10 to 1000 where rather clear power law behavior is found. The applied procedure is described in detail in the book of Hergarten (2002).

Figure 4 gives the exponents obtained for $k=1$ on grids of different sizes from $128 \times 128$ to $2048 \times 2048$ masses. However, when comparing results obtained from grids of different sizes, it should be taken into account that the mean number of events per unit time depends on the grid size. The mean recurrence time $T$ of earthquakes (regardless of their size) decreases from $T=1.8 \times 10^{-4}$ on the $128 \times 128$ grid to $T=3.5 \times 10^{-6}$ on the $2048 \times 2048$ grid. We therefore use $T$ as a time scale, i.e. consider the rescaled time $\frac{\tau}{T}$. Furthermore, the power-law exponent $b$ slightly depends on the grid size, so that we consider the rescaled property $\frac{b}{b_{0}}$ where $b_{0}$ is the exponent obtained for $\tau=0$ on the considered lattice. The effect of the finite rupture propagation time $\tau$ becomes weaker if the grid size increases. However, the lattice sizes considered in this study are not sufficient to ascertain whether the effect completely vanishes in the limit of infinite lattice size. The decreasing distance of adjacent curves in Fig. 4 suggests that the effect may persist even for infinite grid size.

In general, results obtained in the strongly nonconservative regime (large values of $k$ ) should be considered

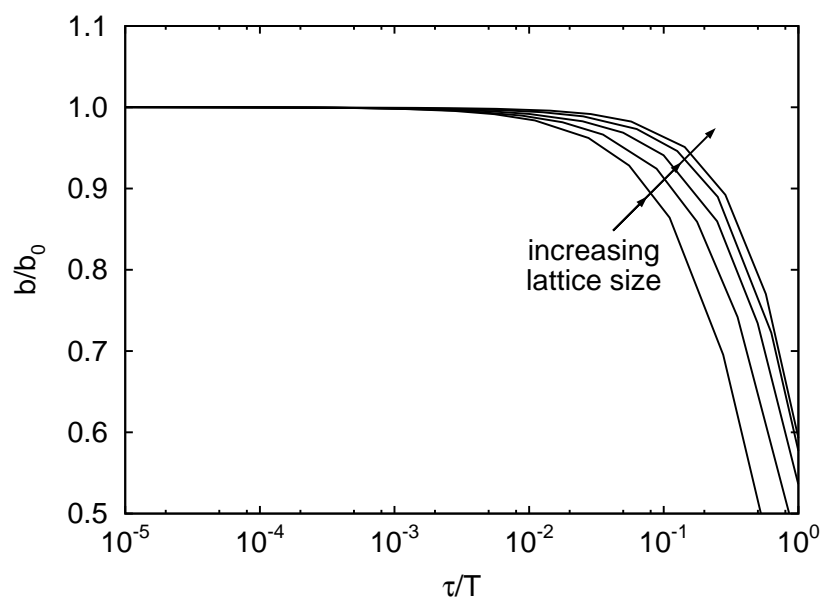

Fig. 4. Dependence of the power-law exponent $b$ of $\tau$ obtained for $k=1$ and lattice sizes from $128 \times 128$ to $2048 \times 2048$ masses (from left to right).

with some caution. A large number of events must be simulated in order to approach the critical state until transient components have died out. For $k=2$, we have skipped $2.5 \times 10^{10}$ events before the analyzed sequence was taken, for $k=4$ this number was ten times higher. Although transient components seem to be weaker under the free boundary conditions used here compared to the widely used rigid-frame boundary conditions (Hergarten, 2002), the curves shown in Fig. 4 are not entirely time-independent. By skipping up to $10^{12}$ events we still found a variation in the curves which slightly exceeds the line width, but not a systematic trend toward one direction. We therefore conjecture that the curves are exposed to some time-dependent noise, but appear to be free of systematic variation through time. However, a more detailled analysis of transient components would go beyond the scope of this paper.

We now make a rough estimate on realistic values of $\tau$ in order to assess this result. Let us, e.g. consider the $1024 \times 1024$ lattice as a representation of a large fault of $100 \times 100 \mathrm{~km}^{2}$, so that grid spacing corresponds to $0.1 \mathrm{~km}$. If we assume that the speed of rupture propagation is at least $1 \frac{\mathrm{km}}{\mathrm{s}}$ (which is rather low), we obtain $\tau \leq 0.1 \mathrm{~s}$. Remember that one time unit in the OFC model is the time span needed to reload a completely relaxed region to the threshold of instability. Therefore, one time unit should correspond to at least 100 years even for very active faults, so that $\tau<4 \times 10^{-11}$ in the units of the OFC model. In combination with the mean recurrence time $T \approx 8 \times 10^{-6}$ obtained for this lattice, we arrive at $\frac{\tau}{T}<5 \times 10^{-4}$ which is in the range where the effect of $\tau$ is negligible (Fig. 4).

However, when transferring this result to real seismicity it should be kept in mind that clustering of earthquake sequences in the OFC model is weaker than in nature (Helmstetter et al., 2004); in real seismicity there are more earthquakes immediately after a mainshock which probably 
cannot be distinguished from the mainshock. The effect of the rupture propagation velocity on the b-value may therefore in nature be stronger than found in this model study.

Let us now consider our results in view of a study on the effect of finite numerical precision recently published (Drossel, 2002). In this study, it was found that the event size distribution in the OFC model depends on the floating point precision. Single and double precision were compared with the result that double precision yields less large events and, in particular, more events of size 1 than single precision.

Numerical precision and finite rupture propagation time may be closely related to each other. Let us assume that two earthquakes take place in the OFC model with infinite precision within a time interval of length $\Theta$. If the numerical precision is not as good as $\Theta$, the two events will take place simultaneously. The same happens in our model with finite rupture propagation time if the duration of the first earthquake is larger than $\Theta$. Thus, the numerical precision is related to the rupture propagation time $\tau$ at least by its order of magnitude.

Let us assume a numerical precision of 8 digits for single precision floating point arithmetics. Since the forces $F_{i}$ of nearly unstable masses are close to unity, the accuracy of these forces is about $10^{-8}$. Coming back to Fig. 4 and remember that $T=3.5 \times 10^{-6}$ on the $2048 \times 2048$ grid, we recognize that $\frac{\tau}{T}$ should be in the order of magnitude of $10^{-3}$. This is the range where the effect of the finite rupture propagation time just becomes visible. Thus, a slight difference between single and double precision is not surprising in view of our study on finite rupture propagation times.

In the study on numerical precision, it was suspected that the dominance of small events becomes stronger in the limit of infinite precision, so that finally only events of size $1 \mathrm{oc}-$ cur. The results shown in Fig. 4 seem not to confirm this suspicion since the curves converge towards one for small values of $\tau$. However, we have additionally analyzed the number of events involving only one mass as a function of $\tau$ explicitly without any clear result whether this number is limited for $\tau \rightarrow 0$, so that this question can only be answered with the help of simulations of higher accuracy than double precision. On the other hand, this is a mainly theoretical question since realistic values of the earthquake duration correspond to a precision somewhere between single and double.

However, the results on the effect of numerical precision published by Drossel (2002) were derived from simulations in the strongly nonconservative regime $\alpha_{i} \leq 0.1$, which means $k \geq 6$. Fig. 5 presents our results obtained for different values of $k$ from 0.5 to 4 and a lattice of $512 \times 512$ masses. Obviously, the effect of the finite rupture propagation time extends towards smaller values of $\frac{\tau}{T}$ if $k$ increases. This confirms the strong difference between single and double precision found by Drossel (2002), but still has no effect if realistic values of $\tau$ are used.

Therefore, using the original OFC model with instantaneous relaxation and double precision arithmetics is appropriate at least for $k \leq 4$; there is neither a need to regard a finite rupture propagation time, nor a need to improve accu-

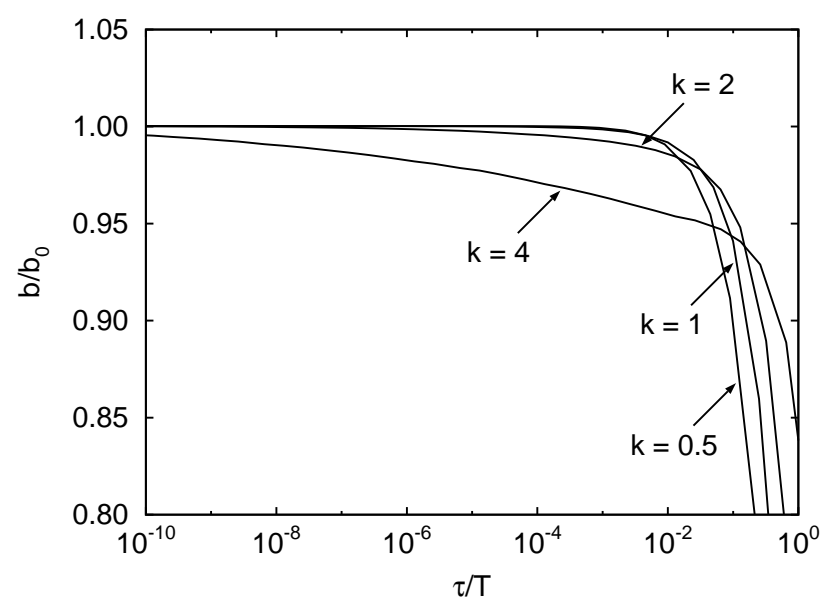

Fig. 5. Dependence of the power-law exponent $b$ of $\tau$ for $k=0.5,1$, 2 and 4.

racy beyond double precision. Using single precision may have a visible effect if $k \geq 2$, but since most simulations are performed with $k=1$, even single precision should be adequate.

\section{Conclusions}

Our study has shown that introducing a finite earthquake duration affects the results of the OFC model, which is one of the most successful spring-block earthquake models. However, the effect becomes visible only if the rupture propagation is quite slow, so that the assumption of instantaneous relaxation made in the original OFC model is appropriate with respect to application, although the effect may be stronger in nature than in the OFC model. A finite numerical precision has similar effects as a finite rupture propagation time, and we found that realistic values correspond to a floating point precision somewhere between single and double. Thus, the question for the model's behavior in the limit of infinite numerical precision is a rather theoretical matter without consequences for model applications in seismology.

Acknowledgements. The authors thank A. Helmstetter and an anonymous referee for their helpful suggestions.

Edited by: C. Goltz

Reviewed by: A. Helmstetter and another referee

\section{References}

Bak, P.: How Nature Works - the Science of Self-Organized Criticality, Copernicus, Springer, Berlin, Heidelberg, New York, 1996.

Bak, P., Tang, C., and Wiesenfeld, K.: Self-organized criticality, An explanation of 1/f noise, Phys. Rev. Lett., 59, 381-384, 1987. 
Brown, S. R., Scholz, C. H., and Rundle, J. B.: A simplified springblock model of earthquakes, Geophys. Res. Lett., 18, 215-218, 1991.

Burridge, R. and Knopoff, L.: Model and theoretical seismicity, Bull. Seismol. Soc. Am., 57, 341-371, 1967.

Carlson, J. M., Langer, J. S., and Shaw, B. E.: Dynamics of earthquake faults, Rev. Mod. Phys., 66, 657-670, 1994.

Corral, A. and Paczuski, M.: Avalanche merging and continuous flow in a sandpile model, Phys. Rev. Lett., 83, 572-575, 1999.

Drossel, B.: Complex scaling behavior in nonconserved selforganized critical systems, Phys. Rev. Lett., 89, 238 701, 2002.

Grassberger, P.: Efficient large-scale simulations of a uniformly driven system, Phys. Rev. E, 49, 2436-2444, 1994.

Gutenberg, B. and Richter, C. F.: Seismicity of the Earth and Associated Phenomenon., Princeton University Press, Princeton, 2nd edn., 1954.

Hamon, D., Nicodemi, M., and Jensen, H. J.: Continuously driven OFC: A simple model of solar flare statistics, Astronomy \& Astrophysics, 387, 326-334, 2002.

Helmstetter, A., Hergarten, S., and Sornette, D.: Properties of foreshocks and aftershocks of the nonconservative self-organized critical Olami-Feder-Christensen model, Phys. Rev. E, 70, 046120, 2004.

Hergarten, S.: Self-Organized Criticality in Earth Systems, Springer, Berlin, Heidelberg, New York, 2002.

Hergarten, S. and Neugebauer, H. J.: Foreshocks and aftershocks in the Olami-Feder-Christensen model, Phys. Rev. Lett., 88, $238501,2002$.

Jensen, H. J.: Self-Organized Criticality - Emergent Complex Behaviour in Physical and Biological Systems, Lecture Notes in Physics, vol. 10, Cambridge University Press, Cambridge, New York, Melbourne, 1998.
Kagan, Y. Y.: Short-term properties of earthquake catalogs and models of earthquake source, Bull. Seismol. Soc. Am., 94, 12071228, 2004.

Kagan, Y. Y. and Knopoff, L.: Stochastic synthesis of earthquake catalogs, J. Geophys. Res., 86, 2853-2862, 1981.

Lise, S. and Paczuski, M.: Scaling in a nonconservative earthquake model of self-organized criticality, Phys. Rev. E, 64, 046111 , 2001a.

Lise, S. and Paczuski, M.: Self-organized criticality and universality in a nonconservative earthquake model, Phys. Rev. E, 63, $036111,2001 b$.

Matsuzaki, M. and Takayasu, H.: Fractal features of the earthquake phenomenon and a simple mechanical model, J. Geophys. Res., 96, 19925-19931, 1991.

Nakanishi, H.: Cellular-automaton model of earthquakes with deterministic chaos, Phys. Rev. A, 41, 7086-7089, 1990.

Olami, Z., Feder, H. J. S., and Christensen, K.: Self-organized criticality in a continuous, nonconservative cellular automaton modeling earthquakes, Phys. Rev. Lett., 68, 1244-1247, 1992.

Rubin, A. M.: Aftershocks of microearthquakes as probes of the mechanics of rupture, J. Geophys. Res., 107, 2142-2157, 2002.

Rundle, J. B. and Jackson, D. D.: Numerical simulation of earthquake sequences, Bull. Seismol. Soc. Am., 67, 1363-1377, 1977.

Turcotte, D. L.: Fractals and Chaos in Geology and Geophysics, Cambridge University Press, Cambridge, New York, Melbourne, 2nd edn., 1997.

Vidale, J. E., Cochran, E. S., Kanamori, H., and Clayton, R. W.: After the lightning and before the thunder; non-Omori behavior of early aftershocks? Eos Trans. AGU, 84(46), Fall Meet. Suppl., Abstract S31A-08, 2003. 